\title{
Estudio observacional prospectivo que evalúa la carga de enfermedad del $\mathrm{HZ}$ en Colombia
}

\author{
Prospective observational study evaluating the \\ disease burden of $\mathrm{HZ}$ in Colombia
}

\author{
Emmanouil Rampakakis, Melissa Stutz • Quebec (Canadá), \\ Homero Antonio Monsanto-Planadeball • Carolina (Puerto Rico), \\ Gonzalo Mejía-Vélez, Andrés Zapata-Cárdenas • Medellín (Colombia), \\ Andrés Eduardo Rico-Carrillo • Bogotá, D.C. (Colombia), \\ Dora Inés Molina de Salazar • Manizales (Colombia), \\ Óscar Enrique Pradilla-Vesga • Bucaramanga (Colombia), \\ KeLLy Deshon Johnson • KenILWORTh (USA)
}

DOI: https://doi.org/10.36104/amc.2021.1636

\section{Resumen}

Introducción: el herpes zóster (HZ) es una enfermedad debilitante que afecta negativamente la calidad de vida (CV). Este estudio buscó describir la carga de la enfermedad por el HZ en Colombia.

Métodos: estudio prospectivo, observacional, de cohorte única realizado en 10 centros clínicos colombianos. Para ser elegibles, los pacientes tenían que ser inmunocompetentes, $\geq 50$ años de edad, y tener un diagnóstico confirmado de $\mathrm{HZ}$ en fase aguda o dolor posherpético. Los resultados del estudio incluyeron el inventario breve del dolor por zóster (ZBPI), la CV evaluada con el cuestionario EQ-5D y la utilización de recursos de atención médica (URAM) debido a HZ. El seguimiento fue de 180 días.

Resultados: se incluyeron 154 pacientes con una media (DE) de la edad de 64.6 (9.6) años. La media (DE) del peor dolor agudo fue 8.2 (2.1), mientras la neuralgia posherpética (dolor por $\mathrm{HZ}$ $\geq 3$ que persistió $\geq 90$ días) fue reportado por $36.5 \%$ de los pacientes. Los predictores significativos del aumento de la carga de dolor fueron la edad avanzada y puntuaciones de peor dolor más altas desde el inicio de la erupción. El aumento en el dolor asociado a HZ estuvo acompañado con reducción significativa en $\mathrm{CV}$, la cual duró aproximadamente 60 días. En términos de la URAM, medicamentos para el $\mathrm{HZ}$ y que fueron recetados a $98.7 \%$ de los pacientes, incluyendo aciclovir en $85.1 \%$ de los pacientes, $79.2 \%$ tuvieron un seguimiento por un médico general, $38.2 \%$ visitó una sala de emergencia y $29.2 \%$ visitó un especialista

Conclusión: HZ está asociado a carga de enfermedad significativa en Colombia, incluyendo dolor, impacto en la CV y URAM. A medida que la población colombiana envejece, se deben implementar estrategias para manejar y/o prevenir de manera más efectiva la carga asociada al HZ. (Acta Med Colomb 2021; 46. DOI: https://doi.org/10.36104/amc.2021.1636).

Palabras clave: herpes zóster, carga de la enfermedad, Colombia, neuralgia posherpética.

\section{Abstract}

Introduction: herpes zoster (HZ) is a debilitating disease with a negative effect on quality of life (QL). This study sought to describe the burden of disease of $\mathrm{HZ}$ in Colombia.

Methods: this was a prospective, observational single-cohort study in 10 Colombian clinical centers. To be eligible, patients had to be immunocompetent, $\geq 50$ years old, and have a confirmed diagnosis of acute phase $\mathrm{HZ}$ or postherpetic pain. The study outcomes included the Zoster Brief Pain Inventory (ZBPI), QL assessed through the EQ-5D questionnaire, and healthcare resource utilization (HCRU) due to HZ. Patients were followed for 180 days.

Results: 154 patients were included with a mean (SD) age of $64.6(9.6)$ years. The mean (SD) worst acute pain was 8.2 (2.1), while postherpetic neuralgia (HZ pain $\geq 3$ which lasted for $\geq 90$ days)
Dres. Emmanouil Rampakakis y Melissa Stutz-: JSS Medical Research Inc., St-Laurent, Quebec (Canadá); Dr. Homero Antonio MonsantoPlanadeball: MSD (IA) LLC, Center for Observational \& Real-World Evidence. Carolina (Puerto Rico); Dr. Gonzalo Mejía-Vélez: Clínica Las Américas, Medellín (Colombia); Dr. Andrés Zapata-Cárdenas: Especialista en Medicina Interna Hospital Universitario San Vicente Fundación, Profesor Asistente Departamento de Medicina Interna Universidad de Antioquia. Medellín (Colombia); Dra. Dora Inés Molina de Salazar: Universidad de Caldas, Manizales (Colombia), Dr. Óscar Enrique Pradilla-Vesga: Fundación Oftalmológica de Santander, Clínica Carlos Ardila Lülle (FOSCAL), Bucaramanga (Colombia), Dr. Kelly Deshon Johnson: Center for Observational \& Real-World Evidence, Kenilworth, NJ, (USA).

Correspondencia: Dr. Homero Antonio Monsanto-Planadeball. Carolina (Puerto Rico). E-mail: homero.monsanto@merck.com Recibido: 19/XII/2019 Aceptado: 04/V/2021 
was reported by $36.5 \%$ of the patients. The significant predictors of increased pain burden were advanced age and higher worst pain score from the onset of the rash. Increased HZ-related pain was associated with a significant reduction in QL, which lasted approximately 60 days. In terms of HCRU, HZ medications were prescribed for $98.7 \%$ of the patients, including acyclovir in $85.1 \%$; $79.2 \%$ were followed by a general practitioner; $38.2 \%$ were seen in the emergency room and $29.2 \%$ consulted a specialist.

Conclusion: $\mathrm{HZ}$ is associated with a significant burden of disease in Colombia, including pain and an impact on QL and HCRU. As the Colombian population ages, strategies should be implemented to more effectively manage and/or prevent the HZ-related burden. (Acta Med Colomb 2021; 46. DOI: https://doi.org/10.36104/amc.2021.1636).

Key words: herpes zoster, burden of disease, Colombia, postherpetic neuralgia

\section{Introducción}

El riesgo estimado de por vida para el desarrollo del herpes zóster (HZ) es de aproximadamente 30\% (1-4). En América del Norte, Asia y Europa, las tasas anuales correspondientes de incidencia de $\mathrm{HZ}$ en la población global se encuentran entre 3-6/1000 personas-años (PYs) $(5,6)$, aumentando a 6-11/1000 PYs mayores de 60 años $(3,5,6)$ y $8-13 / 1000$ PYs mayores de 80 años (5-7). Las estimaciones latinoamericanas de HZ, sin embargo, son escasas. Un estudio brasileño realizado por Castro et al. evaluó, durante 27 meses, las visitas a una clínica de dermatología de emergencia, reportando una tasa de incidencia de aproximadamente 5.6/1000 PYs (8). En Colombia, (9) las estimaciones de incidencia de HZ se limitan a los resultados de un estudio de control de casos realizado en una clínica de oncología, que identificó HZ en $0.65 \%$ de los controles de 11560 pacientes sin enfermedad maligna (9).

Aunque el cuadro clínico varía, el inicio del HZ suele caracterizarse por un pródromo de dolor, seguido de la aparición de una erupción dermatómica unilateral varios días después $(10,11)$. La curación de la erupción demora de dos a tres semanas y el dolor durante esta fase aguda es de moderado a intenso en la mayoría de los pacientes (10-12). En un subconjunto de pacientes, el dolor dura hasta después de la curación de la erupción y se le conoce como neuralgia posherpética (NPH). Se ha determinado que tanto el dolor agudo como el crónico relacionados con el $\mathrm{HZ}$, en los que la incidencia y la intensidad se correlacionan de forma positiva con la edad (4), afectan negativamente la calidad de vida (CV) $(12,13)$. La administración rápida de fármacos antivirales, que se ha demostrado que atenúa la intensidad y la duración del HZ en fase aguda, no se ha relacionado de forma concluyente con una reducción de la incidencia de la NPH (14), aunque se ha determinado que la vacunación profiláctica para el zóster, reduce tanto la carga de la fase aguda de la enfermedad como la incidencia de la NPH (15). En un estudio por Baxter et al. se determinó que la eficacia a largo plazo de una vacuna profiláctica para el zóster es $67.5 \%$, después del primer año posterior a la vacunación, con una reducción a 47.2 y $31.8 \%$ después de dos y ocho años, respectivamente, que da como resultado una eficacia general de la vacuna de $49.1 \%$ (16).

En la actualidad, no se dispone de datos sobre la presentación clínica del HZ, su impacto en el bienestar del paciente, así como su manejo en la vida real en Colombia. Los objetivos del presente estudio fueron describir la carga en la vida real del HZ en Colombia, incluyendo el dolor relacionado con el HZ, la utilización de recursos y el impacto en la calidad de vida.

\section{Métodos}

\section{Diseño del estudio}

Este fue un estudio prospectivo, observacional, de cohorte única realizado en 10 centros clínicos en Colombia, cinco especializados en medicina interna (uno en Bogotá, uno en Cali, uno en Manizales y dos en Medellín), dos en medicina de familia/general (uno en Bogotá, uno en San Gil), y uno en cada uno de las siguientes: neurología (en Bucaramanga), endocrinología (en Barranquilla) y dermatología (en Bogotá). El propósito del estudio fue calcular la carga de la enfermedad debido al HZ tanto en los casos incidentes, definidos como los pacientes con un episodio de $\mathrm{HZ}$ de una duración de menos de siete días respecto de la inscripción en el estudio, como los prevalentes, definidos como los pacientes que se inscribieron después de este margen de siete días, pero dentro de los 90 días del inicio de la erupción.

El estudio implicó un riesgo mínimo según la Resolución 8430 de 1993, fue revisado y aprobado por los organismos locales apropiados de revisión de la ética, y se realizó de acuerdo con las Directrices de la Conferencia Internacional de Armonización para las Buenas Prácticas Clínicas y los principios de la Declaración de Helsinki.

\section{Población del estudio}

Se inscribió a pacientes con HZ desde el consultorio de sus médicos tratantes por un periodo de 18 meses. Para ser elegible para participar, los pacientes tenían que: 1 . tener un diagnóstico confirmado por el médico de $\mathrm{HZ}$ en fase aguda o dolor posherpético, con un diagnóstico principal dentro de 90 días antes de la visita de inscripción, 2. tener al 
menos 50 años al momento del inicio de la erupción de HZ, 3 . ser capaz de comprender y completar los cuestionarios del estudio, y 4. poder dar su consentimiento informado. Se excluyó del estudio a los pacientes inmunocomprometidos, según definido a continuación: 1 . presencia de enfermedad inmunosupresora, 2. uso de corticosteroides (excepto tópico o inhalado de forma intermitente), 3. recibir terapia inmunosupresora o citotóxica, o 4. presencia de cualquier enfermedad neoplásica, excepto por cáncer de piel localizado y otros tumores malignos sólidos estables sin terapia inmunosupresora o citotóxica.

\section{Calendario y evaluaciones del estudio}

La duración del seguimiento fue de seis meses, independientemente de la fase de $\mathrm{HZ}$ en la inscripción. En el caso de los pacientes elegibles que dieron su consentimiento informado, se registraron en la inscripción (día 0) las características sociodemográficas y de referencia de la enfermedad. Se evaluó la carga de enfermedad con las evaluaciones que se describen a continuación.

En la inscripción, se evaluó la calidad de vida (CV) anterior al episodio de HZ con el Euro-QoL (EQ-5D) (15), un instrumento consolidado de $\mathrm{CV}$ diseñado para completarse automáticamente. La puntuación del índice del EQ-5D se calcula mediante la evaluación de cinco dimensiones, específicamente la movilidad, el cuidado personal, las actividades regulares, el dolor/la molestia y la ansiedad/la depresión. Estas dimensiones califican el estado de salud del paciente en cinco niveles diferentes: sin problemas, problemas leves, problemas moderados, problemas graves y problemas extremos. La percepción general de la salud también se calificó en la escala análoga visual (VAS) de 100 mm que acompañó al EQ-5D, en la que una puntuación más alta indica una mejor $\mathrm{CV}$. Aunque tenía como objetivo la recolección prospectiva de datos, un estudio anterior de Wilson et al. concluyó que, a pesar del riesgo de un pequeño sesgo ascendente, el uso retrospectivo del EQ-5D es más adecuado que el uso de normas de población para evaluar la calidad de vida antes del inicio de una lesión aguda/enfermedad (18).

También se administró el cuestionario inicial de impacto del zóster (IZIQ) (19) para recopilar información relacionada con la carga del dolor prodrómico en una escala de 0-10, donde 0 representaba "nada de dolor" y 10 "el peor dolor posible". Una puntuación de dolor de 7 de 10 o más se consideró intensa.

El seguimiento consistió en entrevistas telefónicas, realizadas los días 30, 60, 120 y 150; también se programaron entrevistas en el consultorio el día 90 y el día 180 (visita de fin del estudio). En todas las visitas, incluida la inscripción (día 0), los pacientes completaron los siguientes cuestionarios administrados por ellos mismos o el entrevistador: el inventario breve del dolor por zóster (ZBPI) (19), calificado según el IZIQ que se describió anteriormente, y para evaluar la CV actual se utilizaron la información relacionada con el dolor en fase aguda (actual y peor/menor/promedio en las últimas 24 horas) y el índice del EQ-5D y de la VAS.

También se evaluó la utilización de recursos de atención médica (URAM) con un cuestionario simple que les preguntaba a los pacientes si habían buscado atención médica adicional debido al HZ desde la última visita del estudio/ entrevista telefónica.Además, se evaluó en todas las visitas el tratamiento concomitante de $\mathrm{HZ}$ y la incidencia de la NPH, definida como una puntuación en el ZBPI de "dolor en las últimas 24 horas" relacionado con el $\mathrm{HZ}$ de $\geq 3$ que dure 90 días después del inicio de los síntomas de HZ.

Sólo para los casos de HZ incidente, también se les solicitó a los pacientes que completaran los cuestionarios del ZBPI, el EQ-5D y la URAM para los días 7, 14 y 21 y los enviaran por correo o los llevaran a la próxima visita del estudio en el consultorio el día 90.

\section{Análisis estadísticos}

Se realizaron análisis en la cohorte general del estudio. Se elaboraron estadísticas descriptivas para todas las variables del estudio, que incluyeron la media, la mediana y el intervalo de confianza (CI) de $95 \%$ de la media para variables continuas, así como distribuciones de frecuencia para las variables categóricas. La puntuación del índice del EQ-5D se calculó según los pesos de preferencia de América Latina (20).

Se estimó la carga del dolor debido al HZ desde el área bajo la curva (ABC) de la medida del ZBPI de "peor dolor en las últimas 24 horas" frente al tiempo desde el inicio de los síntomas, donde el inicio de los síntomas se refiere a erupción o dolor, lo que ocurriera primero. Los predictores de la carga del dolor se identificaron usando modelos mixtos multivariables con medidas repetidas, con el ajuste de la duración del inicio de los síntomas del HZ informado en la inscripción y teniendo en cuenta las siguientes covariables según lo informado en la inscripción: la edad al momento del inicio de los síntomas de HZ; el nivel educativo; el estatus de empleo; los medicamentos para el HZ; la cantidad de lesiones en la inscripción; el HZ incidente vs. prevalente y las peores puntuaciones de dolor prodrómico y general. Se utilizó la selección retroactiva de variables para derivar el modelo parsimonioso.

Todos los análisis se realizaron usando software de SAS versión 9.4 (SAS Institute Inc., Cary, NC).

\section{Resultados \\ Características sociodemográficas}

La Tabla 1 presenta las características sociodemográficas de la cohorte del estudio al momento de la inscripción. La edad media (DE) general fue 64.6 (9.6) años, el 35.7\% de los pacientes tenían entre 50 y 59 años, el $38.3 \%$ entre 60 y 69 años y el $26.0 \%$ más de 70 años. La mayoría de los pacientes fueron femeninos $(62.3 \% ; \mathrm{n}=96) \mathrm{y}$ de origen hispano $(65.6 \% ; n=101)$. Con respecto a la educación, poco más de $55 \%(\mathrm{n}=87)$ de los pacientes informaron un nivel de escolaridad primario o secundario. El 20.8\% ( $\mathrm{n}=$ 
Tabla 1. Características sociodemográficas de los pacientes.

\begin{tabular}{|c|c|}
\hline Parámetro & $\begin{array}{l}\text { Cohorte total } \\
\qquad N=154\end{array}$ \\
\hline Edad al inicio de los síntomas, años, media (DE) & $64.6(9.6)$ \\
\hline \multicolumn{2}{|l|}{ Rango de edad al inicio de los síntomas, años, n (\%) } \\
\hline $50-59$ & $55(35.7)$ \\
\hline $60-69$ & $59(38.3)$ \\
\hline$\geq 70$ & $40(26.0)$ \\
\hline Género, femenino, n (\%) & $96(62.3)$ \\
\hline \multicolumn{2}{|l|}{ Raza, n (\%) } \\
\hline Caucásica & $24(15.6)$ \\
\hline Negra & $1(0.6)$ \\
\hline Hispana & $101(65.6)$ \\
\hline Otra & $28(18.2)$ \\
\hline \multicolumn{2}{|l|}{ Nivel más alto de educación, n (\%) } \\
\hline Primario & $56(36.4)$ \\
\hline Secundario & $31(20.1)$ \\
\hline Grado asociado & $23(14.9)$ \\
\hline Universitario & $25(16.2)$ \\
\hline Posgrado & $19(12.3)$ \\
\hline \multicolumn{2}{|l|}{ Estatus de empleo actual, n (\%) } \\
\hline Tiempo completo & $32(20.8)$ \\
\hline Tiempo parcial & $12(7.8)$ \\
\hline Retirado & $55(35.7)$ \\
\hline Desempleado & $9(5.8)$ \\
\hline Sin trabajar debido a incapacidad & $2(1.3)$ \\
\hline A cargo del hogar a tiempo completo & $30(19.5)$ \\
\hline Otra & $14(9.1)$ \\
\hline \multicolumn{2}{|l|}{ Estatus de inmunidad, n (\%) } \\
\hline Normal & $107(69.5)$ \\
\hline Comprometida $^{1}$ & $47(30.5)$ \\
\hline \multicolumn{2}{|c|}{$\begin{array}{l}\text { 'Comprometida incluye lo siguiente: insuficiencia vitamínica/mineral }(\mathrm{n}=1) \text {; estilo } \\
\text { de vida estresante/agotador }(\mathrm{n}=18) \text {; consumo excesivo de cigarrillos }(\mathrm{n}=8) \text {; abuso } \\
\text { de alcohol }(\mathrm{n}=3) \text {; uso intermitente de corticosteroides tópicos/inhalados }(\mathrm{n}=2) \text {; } \\
\text { trastorno autoinmune }(\mathrm{n}=8) \text {; trastorno alérgico }(\mathrm{n}=20) \text {; tumor maligno estable }(\mathrm{n} \\
=1) \text {. Los pacientes pueden haber informado más de una condición inmunológica. }\end{array}$} \\
\hline
\end{tabular}

32) de los pacientes informó empleo a tiempo completo, un $7.8 \%(\mathrm{n}=12)$ informó empleo a tiempo parcial y $35.7 \%$ ( $\mathrm{n}$ $=55$ ) estaba retirado.

\section{Características del herpes zóster}

Las características de la enfermedad de HZ se resumen en la Tabla 2. La duración media (DE) de los síntomas de HZ antes de la visita de inscripción fue 35.9 (24.0) días y se clasificó a $93.5 \%(n=144)$ de los casos de HZ como prevalentes. La duración media (DE) desde el inicio de la erupción en la inscripción fue 21.3 (17.6) días. Entre los pacientes que informaron erupción en la inscripción $(\mathrm{n}=$ 87; 56.5\%), la zona más común afectada de dermatoma principal fue la torácica $(\mathrm{n}=40 ; 26.0 \%)$ y la cantidad de lesiones presentes en los dermatomas principales y adyacentes se dividía principalmente entre 1-20 lesiones
Tabla 2. Características de la enfermedad.

\begin{tabular}{|c|c|}
\hline Parámetro & $\begin{array}{c}\text { Cohorte total } \\
\mathrm{N}=154\end{array}$ \\
\hline Duración desde el inicio de los síntomas de HZ, días, media (DE) & $35.9(24.0)$ \\
\hline Grupo de caso de $\mathrm{HZ}, \mathrm{n}(\%)$ & \\
\hline Incidente & $10(6.5)$ \\
\hline Prevalente & $144(93.5)$ \\
\hline Duración desde el inicio de la erupción, días, media (DE) & $21.3(17.6)$ \\
\hline Erupción por HZ presente en la inscripción, sí, n (\%) & $87(56.5)$ \\
\hline Zona afectada de dermatoma principal, n (\%) & \\
\hline Nervio trigémino & $17(11.0)$ \\
\hline Cervical & $16(10.4)$ \\
\hline Torácica & $40(26.0)$ \\
\hline Sacra & $4(2.6)$ \\
\hline Lumbar & $10(6.5)$ \\
\hline No aplica & $67(43.5)$ \\
\hline $\begin{array}{l}\text { Cantidad de lesiones en los dermatomas principales y adya- } \\
\text { centes, } \mathrm{n}(\%)\end{array}$ & \\
\hline Ninguna & $2(1.3)$ \\
\hline $1-20$ & $34(22.1)$ \\
\hline $11-20$ & $24(15.6)$ \\
\hline $21-50$ & $22(14.3)$ \\
\hline $51-100$ & $5(3.2)$ \\
\hline No aplica & $67(43.5)$ \\
\hline Dolor prodrómico, sí, n (\%) & $109(70.8)$ \\
\hline $\begin{array}{l}\text { Duración del dolor antes del inicio de la erupción, días, } \\
\text { media (DE) }\end{array}$ & $5.2(8.8)$ \\
\hline Peor dolor, media $(\mathrm{DE})^{1}$ & $7.2(2.7)$ \\
\hline Dolor promedio, media (DE) ${ }^{1}$ & $5.8(2.6)$ \\
\hline $\begin{array}{l}\text { Dolor asociado al HZ desde la aparición de la erupción por } \\
\mathrm{HZ} \text {, sí, n (\%) }\end{array}$ & $147(95.5)$ \\
\hline Duración del dolor, días, media (DE) & $17.6(15.0)$ \\
\hline Peor dolor, media $(\mathrm{DE})^{1}$ & $8.2(2.1)$ \\
\hline Dolor promedio, media (DE) ${ }^{1}$ & $6.0(2.0)$ \\
\hline Tratamiento iniciado para el HZ, sí, n (\%) & $150(98.7 \%)$ \\
\hline Medicamento para el HZ, n (\%) & \\
\hline Aciclovir & $131(85.1)$ \\
\hline Aciclovir (uso dermatológico) & $86(55.8)$ \\
\hline Carbamazepina & $32(20.8)$ \\
\hline Diclofenaco & $25(16.2)$ \\
\hline Acetaminofeno & $71(46.1)$ \\
\hline Pregabalina & $66(42.9)$ \\
\hline Tramadol & $44(28.6)$ \\
\hline Valaciclovir & $25(16.2)$ \\
\hline Pacientes con NPH, n $(\%)^{3}$ & $54(36.5)$ \\
\hline \multicolumn{2}{|c|}{$\begin{array}{l}\text { DE: desviación estándar, HZ: herpes zóster, NPH: neuralgia posherpética. } \\
{ }^{1} \text { Clasificado en una escala del } 1 \text { (sin dolor) al } 10 \text { (el peor dolor imaginable); deter- } \\
\text { minado en la visita de inscripción. } \\
{ }^{2} \text { Los medicamentos para el HZ presentados son los informados por } \geq 15 \% \text { de los } \\
\text { pacientes. } \\
{ }^{3} \text { No se evaluaron seis casos prevalentes que tuvieron un seguimiento de }<90 \text { días } \\
\text { desde el inicio de los síntomas; la proporción se basa en n }=148 \text {. }\end{array}$} \\
\hline
\end{tabular}


$(\mathrm{n}=34 ; 22.1 \%), 11-20$ lesiones $(\mathrm{n}=24 ; 15.6 \%)$ y $21-50$ lesiones $(\mathrm{n}=22 ; 14.3 \%)$.

El $70.8 \%$ de los pacientes informó dolor prodrómico, la duración media (DE) fue de 5.2 (8.8) días antes de la aparición de la erupción y el peor dolor medio (DE) fue de 7.2 (2.7). El 95.5\% de los pacientes presentó dolor agudo por HZ $(n=147)$, con puntuaciones medias para el dolor promedio y el peor dolor de 6.0 (2.0) y 8.2 (2.1) respectivamente. La duración media (DE) del dolor agudo fue de 17.6 (15.0) días. El 36.5\% de los pacientes $(n=54 / 148)$ informó $\mathrm{NPH}$, evaluada en los pacientes con datos disponibles para hasta al menos 90 días posteriores al inicio de los síntomas del HZ. En total, 150 pacientes (el 98.7\%) recibieron una receta para medicamentos para el control de su episodio de HZ (Tabla 2) y aciclovir fue el más común $(\mathrm{n}=131 ; 85.1 \%)$.

\section{Utilización de recursos de atención médica}

En la Tabla 3 se resume la URAM informada por los pacientes en el seguimiento. Las visitas a un médico generalista (MG) fueron el recurso utilizado con mayor frecuencia, el $79.2 \%$ de los pacientes $(\mathrm{n}=122)$ informaron una media (DE) de 2.6 (2.8) visitas cada uno. La proporción de pacientes que informó $\geq 1$ visitas a la sala de emergencias y a especialistas también fue alta: $38.3 \%(n=59$; media [DE]: $1.6[1,0]$ visitas $)$ y $29.2 \%(n=45$; media [DE]: $2.6[3,1]$ visitas) respectivamente. En general, el $14.3 \%(n=22)$ de los pacientes fue hospitalizado por su episodio de $\mathrm{HZ}$, con una duración media (DE) de la estadía entre los usuarios de 5.0 (4.7) días. Aunque el uso de servicios de enfermería ( $\mathrm{n}=10$; $6.5 \%)$ y psicoterapia/rehabilitación $(n=4 ; 2.6 \%)$ fue poco frecuente, la cantidad media (DE) de servicios por usuario fue elevada: 13.1 (27.5) y 21.0 (27.8) respectivamente.

\section{Dolor en el tiempo}

La Figura 1A presenta el dolor relacionado con el HZ en el tiempo, evaluado con las preguntas del ZBPI que analizan el "dolor promedio" y el "peor dolor en las últimas 24 horas" entre los pacientes con una puntuación de dolor de 1 o más (0 representaba que no había dolor). La puntuación de dolor promedio de ZBPI medio (DE) tuvo su pico el día 7 , 5.2 (2.2), y disminuyó gradualmente en el tiempo hasta 2.6 (1.7) el día 120. Se observó un aumento leve hacia el día 150 y el día 180, que regresó a una puntuación promedio de 3.1 en ambas visitas. Se observó una tendencia similar para el peor dolor del ZBPI, con una puntuación elevada de
Tabla 3. Utilización de recursos de atención médica relacionados con el HZ.

\begin{tabular}{|l|c|c|}
\hline \multicolumn{1}{|c|}{ Tipo de URAM } & \multicolumn{2}{c|}{ Cohorte total } \\
& N=154 \\
\hline & N (\%) & $\begin{array}{c}\text { Media } \\
(\text { DE })^{1}\end{array}$ \\
\hline Médico general (MG) & $122(79.2)$ & $2.6(2.8)$ \\
Sala de emergencias (SE) & $59(38.3)$ & $1.6(1.0)$ \\
Hospitalización & $22(14.3)$ & $5.0(4.7)^{2}$ \\
Servicios de enfermería & $10(6.5)$ & $13.1(27.5)$ \\
Fisioterapista/rehabilitación & $6(3.9)$ & $21.0(27.8)$ \\
Psiquiatra/psicólogo/consejero & $4(2.6)$ & $1.5(0.6)$ \\
Especialista & $45(29.2)$ & $2.6(3.1)$ \\
\hline${ }^{1}$ Cantidad de contactos de atención médica entre usuarios de los respectivos recursos \\
de atención médica. \\
${ }^{2}$ Cantidad de días de hospitalización.
\end{tabular}

6.4 (2.4), informada el día 7, que se redujo a 4.1 (2.3) el día 180 , y que tuvo un aumento leve a 4.6 (2.4) y 5.0 (2.6) los días 150 y 180 , respectivamente.

Se identificaron predictores de la carga de dolor, calculados a partir del área bajo la curva del "peor dolor en el tiempo desde el inicio de la erupción por HZ", a partir de los análisis de modelos mixtos multivariables (Tabla 4). En un modelo reducido se identificó al aumento en la edad como un predictor positivo significativo de la carga de dolor $(\mathrm{p}<0.05)$ (Tabla 4); una puntuación del "peor dolor del ZBPI desde la erupción por HZ" $\geq 7$, evaluado en la inscripción, también se relacionó de forma significativa con una mayor carga de dolor en comparación con las puntuaciones de 0-6 ( $\mathrm{p}<0.001)$.

\section{Calidad de vida en el tiempo}

La CV en el tiempo se presenta en la Figura 1B (VAS del EQ-5D) y en la Figura 1C (índice del EQ-5D; pesos en América Latina). Con respecto al primero, la media (DE) de la calidad de vida autocalificada previa al HZ, entre los pacientes con respuesta, fue de 89.3 (13.1) y se redujo a 73.7 (21.1) en la visita inicial y a 70.5 (19.9) el día 7. Posteriormente, se observaron aumentos constantes en el tiempo hasta el día 180, en el que se informó una CV prácticamente previa al HZ de 88.6 (14.4) mm. La puntuación del índice del EQ-5D entre los pacientes con respuesta fue de una media (DE) de $0.9(0.2)$ antes del inicio del HZ y se redujo a 0.7 (0.2) tanto en la visita de inscripción como el día 7. Se volvió a alcanzar una puntuación del índice de la CV previa

Tabla 4. Modelo mixto de medidas repetidas*: predictores de carga de dolor acumulada.

\begin{tabular}{|c|c|c|c|c|}
\hline Predictor & Estimado & DE & IC del $95 \%$ & Valor de $\mathbf{p}$ \\
\hline Edad al inicio de los síntomas de HZ, años & 3.85 & 1.93 & $0.03 ; 7.67$ & 0.048 \\
\hline Peor puntuación de dolor de ZBPI desde la erupción de HZ: intenso ( $\geq 7$ ) vs. leve/moderado (0-6) & 251.29 & 44.01 & $164.3 ; 338.2$ & $<0.001$ \\
\hline \multicolumn{5}{|c|}{$\begin{array}{l}\text { DE: desviación estándar; IC: intervalo de confianza; ZPBI: inventario breve del dolor por zóster; HZ: herpes zóster. } \\
\text { * Modelo reducido luego de la selección retroactiva de variables y del ajuste para la duración del inicio de los síntomas en la inscripción. Las variables que se consideraron fueron las } \\
\text { siguientes: el tiempo desde el inicio de los síntomas de HZ y la edad en ese momento; el nivel educativo; el estatus de empleo; los medicamentos para el HZ; la cantidad de lesiones en } \\
\text { la selección; el HZ incidente vs. prevalente; la duración del inicio de los síntomas en la inscripción; las peores puntuaciones de dolor prodrómico y general. }\end{array}$} \\
\hline
\end{tabular}




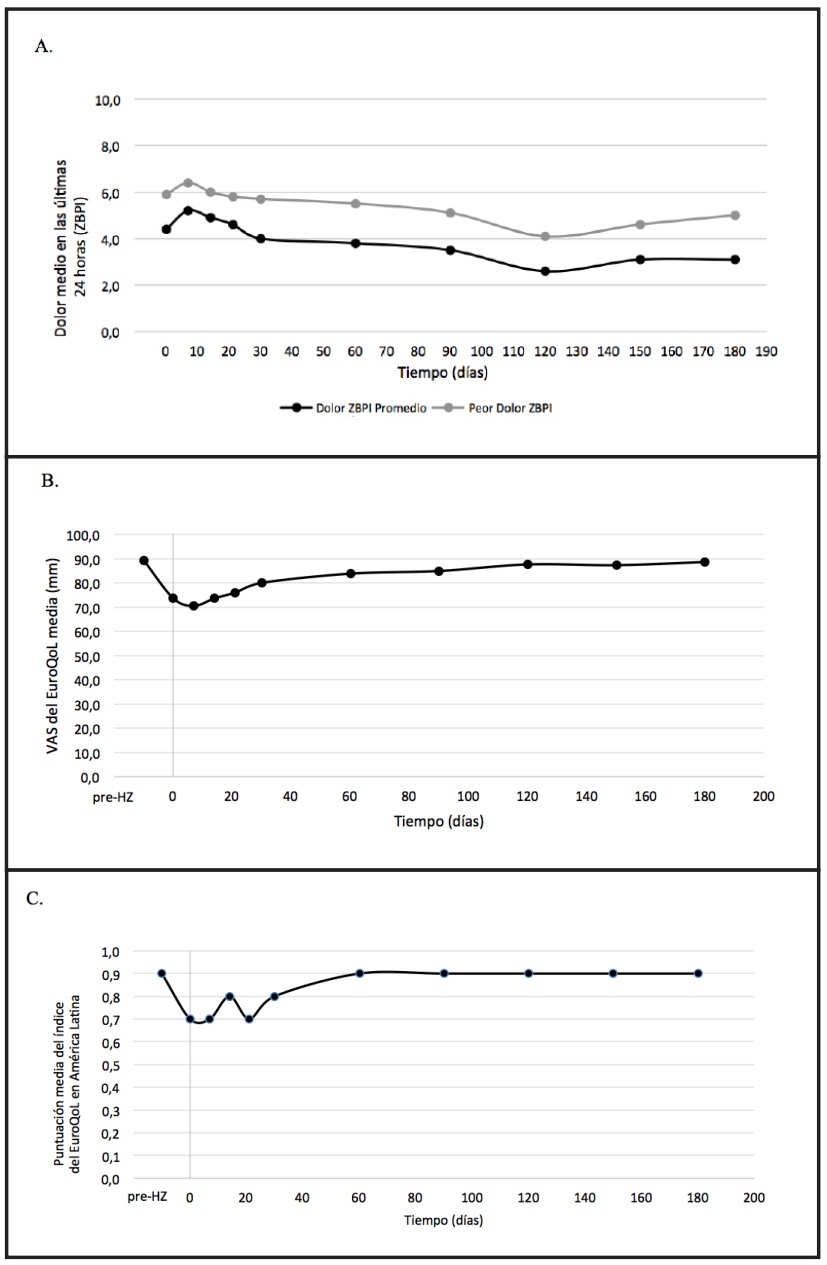

Figura 1. Dolor relacionado con el HZ, calidad de vida y carga de enfermedad en el tiempo. A. Puntuación promedio y peor puntuación de dolor relacionado con el HZ en las últimas 24 horas en el tiempo. B. Puntuaciones de la VAS del EuroQoL-5D en el tiempo. C. Puntuación del índice del EuroQoL-5D (pesos en América Latina) en el tiempo.

al HZ de 0.9 (0.2) el día 60 y se mantuvo constante hasta el final del estudio.

\section{Discusión}

La carga clínica y económica del HZ se ha estudiado previamente en otras regiones de América Latina (21-25); sin embargo, hasta donde tenemos conocimiento, este es el primer informe sobre la carga del HZ en un contexto colombiano. En general, los hallazgos de este estudio prospectivo, observacional indican que la carga del $\mathrm{HZ}$ en Colombia es alta, afecta la calidad de vida, promueve la utilización de recursos y resulta en tasas elevadas de dolor posherpético, prodrómico y agudo. Debido a que $16.6 \%$ de la población colombiana tiene $\geq 55$ años de edad (26), esta carga continuará aumentando en el tiempo, a menos que se implementen estrategias de tratamiento adecuadas.

Aunque se reclutaron pacientes en diferentes estadios de los episodios actuales de HZ, la URAM durante el seguimiento fue elevada; aproximadamente $80 \%$ de los pacientes visitaron un médico general, 38 y $30 \%$ adicionales informaron visitas a salas de emergencias y especialistas, respectivamente. Esto se observó a pesar de que se recetó tratamiento a más de $98 \%$ de los pacientes para los episodios de HZ y coincide con las tasas observadas y la intensidad del dolor relacionado con el HZ que se analizan a continuación. Un hallazgo interesante de este estudio fue el uso del aciclovir tópico informado por más de $50 \%$ de los pacientes; dado que el uso de antivirales tópicos no se recomienda en la actualidad para el tratamiento del HZ (2), este resultado refleja el tratamiento real del HZ en Colombia y puede alertar acerca de una necesidad insatisfecha en relación con la educación de los médicos y el conocimiento respecto de las modalidades de tratamiento de acuerdo con las mejores prácticas.

En general, aproximadamente $71 \%$ de los pacientes informaron dolor prodrómico en el enrolamiento, con notificaciones de "dolor desde la aparición de la erupción" en más de $95 \%$ de los casos. En el peor de los casos, el dolor se consideraba intenso (puntuación promedio $\geq 7$ ), tanto para las fases de dolor prodrómico como agudo. Asimismo, más de $36 \%$ de los pacientes informó NPH. Este cálculo de la NPH se ajusta a $38.6 \%$ informado previamente por Rampakakis et al., en un análisis agrupado de pacientes argentinos, mexicanos y brasileños con $\mathrm{HZ}$ (23), aunque es más alto que los cálculos mexicanos y argentinos de aproximadamente $11 \%(24,25)$. La variación en las tasas observadas de NPH puede atribuirse a lo siguiente: 1) para este estudio, más de 90\% de los pacientes presentaban $\mathrm{HZ}$ prevalente, lo que permitió un marco temporal más prolongado durante el que se podía capturar la NPH, mientras que el cálculo de la Argentina es para una muestra que incluye $40 \%$ de casos incidentes; y 2) la tasa de incidencia mexicana se basa en los datos informados al sistema nacional de información de salud que brinda información epidemiológica solamente sobre altas hospitalarias.

Con respecto a la carga de dolor (peor dolor en el tiempo desde el inicio de la erupción por HZ), los predictores positivos del aumento de la carga que se identificaron fueron la edad avanzada y las puntuaciones más altas de "peor dolor del ZBPI" desde el inicio de la erupción por HZ. Estos hallazgos coinciden con los informes previos de Tsai et al. (27) y Drolet et al. (28) que evaluaron los predictores de la "gravedad de la enfermedad" (equivalente a la carga de dolor) entre los pacientes con HZ; en ambos estudios se informa una relación positiva entre la gravedad de la enfermedad, el grupo de mayor edad y puntuaciones más altas del peor dolor del ZBPI.

Asimismo, y de acuerdo con la tendencia identificada en los estudios previos de la carga del HZ (22, 25, 27-30), los aumentos del dolor estuvieron acompañados de reducciones en la $\mathrm{CV}$, según la medición mediante el EQ-5D. Las puntuaciones promedio y del peor "dolor del ZBPI en las últimas 24 horas" llegaron al máximo el día 7, en 5.2 y 6.4, respectivamente, y las puntuaciones más bajas de la VAS 
del EQ-5D, de $70.5 \mathrm{~mm}$, se observaron en el mismo punto de evaluación. La mejoría gradual en las puntuaciones de dolor en el tiempo presentó aumentos concurrentes en las puntuaciones de la VAS del EQ-5D para el día 180, en el que la $\mathrm{CV}$ alcanzó los valores prácticamente previos al $\mathrm{HZ}$.

En cuanto a las puntuaciones del índice del EQ-5D, también se observó un impacto negativo en la calidad de vida, con una reducción de 0.9 en el período previo al HZ a 0.7 en la visita de enrolamiento y el día 14, que volvió a los valores iniciales para el día 60. En ligero contraste con lo anterior, en un cálculo reciente de Costa Rica se informó una reducción en las puntuaciones del índice del EQ-5D de 0.8 (previo al HZ) a 0.4 en el inicio, sin recuperación de la $\mathrm{CV}$ previa al $\mathrm{HZ}$ al final del estudio $(0.7 \mathrm{el}$ día 180) (22). Por el contrario, las puntuaciones del índice del EQ-5D informadas en la población argentina con HZ, aunque fueron más bajas en el inicio en comparación con el estudio actual (0.6), lograron alcanzar las puntuaciones índice previas al HZ para el día 21, que se superaron el día 120 (25). Estas variaciones en la CV en el tiempo pueden atribuirse a diferencias entre los estudios en las puntuaciones de dolor: para Costa Rica, los pacientes informaron un promedio inicial más alto y puntuaciones de peor dolor del ZBPI de 6.6 y 7.7, respectivamente, con una reducción a 5.9 y 4.0 el día 180; sin embargo, el peor dolor en el inicio en Argentina fue de 5.5 y aumentó a 0.9 el día 60 y fue de 0.2 el día 180. Esta interpretación también respalda la relación entre el dolor relacionado con el HZ y la reducción en la CV.

Este estudio tiene varias limitaciones que se deben considerar. En primer lugar, la población del estudio incluyó solamente a pacientes de $\geq 50$ años; dado que se sabe que la gravedad de la enfermedad aumenta con la edad, la exclusión de pacientes con HZ más jóvenes puede haber resultado en un sesgo de selección que favorece el reclutamiento de pacientes con HZ ligeramente más avanzado. Este sesgo de selección se agrava aún más por el hecho de que este estudio incluyó a pacientes que buscaban atención médica para el HZ, lo que puede haber resultado en una sobre representación de casos de HZ más graves que en la población en general. Asimismo, ya que no se requería una confirmación de laboratorio de la infección por HZ para la inclusión de pacientes, es posible que se hayan producido errores en la clasificación del HZ. Además, la inclusión de casos prevalentes pudo haber conducido a un sesgo de recuerdo en la evaluación del dolor y la CV antes o en el inicio de la erupción. Sin embargo, dado que la media (DE) de la duración del inicio de la erupción antes del ingreso al estudio fue de tan sólo 21.3 (17.55) días, se puede suponer que es probable que los pacientes notificaran de forma precisa la información relacionada con el $\mathrm{HZ}$ y, por consiguiente, se haya reducido el riesgo del sesgo de recuerdo.

A pesar de estas limitaciones, este estudio brinda información, sobre la carga de la enfermedad por el $\mathrm{HZ}$ en un contexto clínico colombiano. Los resultados no solo indican que el dolor, la URAM y el impacto en la CV debido al HZ son altos, sino también que la edad avanzada y la duración desde el inicio de los síntomas son predictores de la carga de dolor. A medida que las poblaciones de Colombia envejecen, es más importante que nunca considerar estrategias para prevenir la reactivación del HZ, como la implementación de una vacuna para todos, o el aumento del conocimiento sobre el HZ con respecto al reconocimiento de los síntomas, así como las opciones de tratamiento farmacológico. Los resultados de este estudio se pueden usar para evaluar la carga económica del HZ en Colombia.

\section{Referencias}

1. Gershon AA, Gershon MD, Breuer J, Levin MJ, Oaklander AL, Griffiths PD. Advances in the understanding of the pathogenesis and epidemiology of herpes zoster. J Clin Virol. 2010;48 Suppl 1:S2-7.

2. Saguil A, Kane S, Mercado M, Lauters R. Herpes Zoster and Postherpetic Neuralgia: Prevention and Management. Am Fam Physician . 2017;96(10):656-63.

3. Brisson M, Edmunds WJ, Law B, Gay NJ, Walld R, Brownell M, et al. Epidemiology of varicella zoster virus infection in Canada and the United Kingdom. Epidemiology and infection. 2001;127(2):305-14.

4. Yawn BP, Saddier P, Wollan PC, St Sauver JL, Kurland MJ, Sy LS. A population-based study of the incidence and complication rates of herpes zoster before zoster vaccine introduction. Mayo Clinic proceedings. 2007;82(11):1341-9.

5. Johnson BH, Palmer L, Gatwood J, Lenhart G, Kawai K, Acosta CJ. Annual incidence rates of herpes zoster among an immunocompetent population in the United States. BMC infectious diseases. 2015;15:502.

6. Kawai K, Gebremeskel BG, Acosta CJ. Systematic review of incidence and complications of herpes zoster: towards a global perspective. BMJ open. 2014;4(6):e004833.

7. Schmidt-Ott R, Schutter U, Simon J, Nautrup BP, von Krempelhuber A, Gopala K, et al. Incidence and costs of herpes zoster and postherpetic neuralgia in German adults aged $>/=50$ years: A prospective study. The Journal of infection. 2018;76(5):475-82.

8. Castro LGM, Chen S. Zoster: mas frequentes entre jovens que entre idosos (Zoster: more frequent among youngsters than the aged). An Bras Dermatol 1990; 65:129-133.

9. Gaitan M. Herpes Zoster y Cáncer: relaciones entre estas dos enfermedades. Acta Med Colomb. 1981;6:287-93.

10. Gnann JW, Jr., Whitley RJ. Clinical practice. Herpes zoster. The New England journal of medicine. 2002;347(5):340-6.

11. Schmader KE, Dworkin RH. Natural history and treatment of herpes zoster. The journal of pain : official journal of the American Pain Society. 2008;9(1 Suppl 1):S3-9.

12. Katz J, Cooper EM, Walther RR, Sweeney EW, Dworkin RH. Acute pain in herpes zoster and its impact on health-related quality of life. Clin Infect Dis. 2004;39(3):342-8

13. Lukas K, Edte A, Bertrand I. The impact of herpes zoster and post-herpetic neuralgia on quality of life: patient-reported outcomes in six European countries. Z Gesundh Wiss. 2012;20(4):441-51.

14. Bruxelle J, Pinchinat S. Effectiveness of antiviral treatment on acute phase of herpes zoster and development of post herpetic neuralgia: review of international publications. Medecine et maladies infectieuses. 2012;42(2):53-8.

15. Oxman MN, Levin MJ, Johnson GR, Schmader KE, Straus SE, Gelb LD, et al. A vaccine to prevent herpes zoster and postherpetic neuralgia in older adults. The New England journal of medicine. 2005;352(22):2271-84.

16. Baxter R, Bartlett J, Fireman B, Marks M, Hansen J, Lewis E, et al. LongTerm Effectiveness of the Live Zoster Vaccine in Preventing Shingles: A Cohort Study. Am J Epidemiol. 2018;187(1):161-9.

17. EuroQol G. EuroQol--a new facility for the measurement of health-related quality of life. Health Policy. 1990;16(3):199-208.

18. Wilson R, Derrett S, Hansen P, Langley J. Retrospective evaluation versus population norms for the measurement of baseline health status. Health Qual Life Outcomes. 2012;10:68.

19. Coplan PM, Schmader K, Nikas A, Chan IS, Choo P, Levin MJ, et al. Development of a measure of the burden of pain due to herpes zoster and postherpetic neuralgia for prevention trials: adaptation of the brief pain inventory. The journal of pain : official journal of the American Pain Society. 2004;5(6):344-56. 
20. Zarate V, Kind P, Chuang LH. Hispanic valuation of the EQ-5D health states: a social value set for Latin Americans. Value Health. 2008;11(7):1170-7.

21. Kawai K, Rampakakis E, Tsai TF, Cheong HJ, Dhitavat J, Covarrubias AO, et al. Predictors of postherpetic neuralgia in patients with herpes zoster: a pooled analysis of prospective cohort studies from North and Latin America and Asia. Int J Infect Dis. 2015;34:126-31

22. Rampakakis E, Alpizar C, Karellis A, Sampalis JS, Johnson K, Monsanto HA, et al. Measuring the burden of herpes zoster disease in Costa Rica. Acta Medica Costarricense. 2017;59(4):146-52.

23. Rampakakis E, Pollock C, Vujacich C, Toniolo Neto J, Ortiz Covarrubias A, Monsanto H, et al. Economic Burden of Herpes Zoster ("culebrilla") in Latin America. Int J Infect Dis. 2017;58:22-6.

24. Vázquez M CP, Galván F. Varicela y herpes zóster: retos para la salud pública. Salud Pública de México November-December 2017;59(6).

25. Vujacich C dWL, Margari A et al. Dolor, calidad de vida relacionada con la salud y utilización de servicios médicos asociados al herpes zoster en Argentina. Actualizaciones en Sida e Infectologia. 2016;24(92):53-63
26. Hernandez D, Muriel A, Abraira V, Perez G, Porrini E, Marrero D, et al. Renin-angiotensin system blockade and kidney transplantation: a longitudinal cohort study. Nephrol Dial Transplant. 2012;27(1):417-22.

27. Tsai TF, Yao CA, Yu HS, Lan CC, Chao SC, Yang JH, et al. Herpes zosterassociated severity and duration of pain, health-related quality of life, and healthcare utilization in Taiwan: a prospective observational study. Int J Dermatol. 2015;54(5):529-36.

28. Drolet M, Brisson M, Levin MJ, Schmader KE, Oxman MN, Johnson RW, et al. A prospective study of the herpes zoster severity of illness. The Clinical journal of pain. 2010;26(8):656-66.

29. Aunhachoke K, Bussaratid V, Chirachanakul P, Chua-Intra B, Dhitavat J, Jaisathaporn K, et al. Measuring herpes zoster, zoster-associated pain, postherpetic neuralgia-associated loss of quality of life, and healthcare utilization and costs in Thailand. International journal of dermatology. 2011;50(4):428-35.

30. Song H, Lee J, Lee M, Choi WS, Choi JH, Lee MS, et al. Burden of illness, quality of life, and healthcare utilization among patients with herpes zoster in South Korea: a prospective clinical-epidemiological study. Int J Infect Dis. 2014;20:23-30. 